\title{
NEURONAS ESPEJO Y ANTICIPACIÓN EN INTERPRETACIÓN SIMULTÁNEA
}

DOI: http://dx.doi.org/10.12775/RP.2019.005

\begin{abstract}
Zarys treści: Antycypacja to częste zjawisko w tłumaczeniu symultanicznym, a przez część badaczy (Gile, 1995; Chernov 1994, 2004) postrzegana jest jako konieczna z uwagi na syntaktyczne różnice strukturalne w parze językowej, w której pracuje tłumacz. Stoimy na stanowisku, że antycypacja nie może być uważana za strategię, którą tłumacz niejako włącza lub wyłącza w tłumaczeniu. Antycypacja stanowi podstawę reformulacji- jeśli tłumacz nie jest w stanie przewidzieć znaczenia wypowiedzi przed jej zakończeniem, bardzo trudno jest dokonać reorganizacji lub zreformułowania wypowiedzi i oddać w tłumaczeniu słowa, składnię czy znaczenie. W badaniach przekładoznawczych od stosunkowo niedawna pojawity się nowe pola takie jak psycholingwistyka czy kognitywistyka (Ferreira, A. \& Schwieter, J.W., 2015); mogą one rzucić nowe światło na procesy występujące w tłumaczeniu ustnym. W tym miejscu warto wspomnieć o roli, którą odgrywają neurony lustrzane (Rizzolatti et al. 1996), a które mogą służyć wyjaśnieniu zagadnienia, dlaczego w komunikacji - a takowa jest tłumaczenie symultaniczne - zachodzi ciągły i naturalny proces antycypacji.
\end{abstract}

Słowa kluczowe: antycypacja, neurony lustrzane, tłumaczenie symultaniczne

\section{Introducción}

D esde que Rizzolatti et al. descubrieran en 1996 un tipo de neuronas muy especial, a las que denominaron neuronas espejo, el mundo de la neurociencia se ha volcado en buscar explicaciones a las destrezas que activan esas neuronas. Al parecer, pueden estar implicadas en la adquisición de lenguaje, en la representación de imágenes mentales y en la anticipación de intenciones en el movimiento del otro.

La anticipación es materia de interés también en el ámbito de la interpretación simultánea. El intérprete anticipa gran parte del sentido argumental 
del orador durante su tarea. En general, consiste en la facultad de predecir cómo va a continuar el discurso en la lengua de partida (Gile 1995; Chernov 1994 y 2004 citado en Bartlomiejczyk 2008: 118). Chernov (1979, 1981) explicaba que, a pesar de lo complejo de la tarea, se podía activar un modelo de predicción de probabilidades que, a su vez, permitía adelantar o intuir el sentido del discurso, gracias a la redundancia omnipresente en la lengua.

Esta redundancia está presente en lengua natural (Chernov 1979) pero si la situación comunicativa se refiere a un congreso, esta anticipación se suele producir por la preparación previa y los conocimientos lingüísticos del intérprete y por su conocimiento situacional o del mundo (Lederer 1978, 2002; Wills 1978, citados en Bartlomiejczyk 2008: 118).

La anticipación se ha investigado desde varios ángulos. La interpretación simultánea es una actividad que exige un gran esfuerzo cognitivo. En particular, parte de este esfuerzo se debe a que el sentido de los enunciados, la cohesión y coherencia entre las partes del discurso, se van desplegando a medida que avanza el propio discurso. De tal modo, que el intérprete se ve abocado a desarrollar habilidades "adivinatorias" para poder continuar con su tarea.

La investigación sobre esta particularidad cognitiva se ha centrado, por un lado, en la obligatoriedad de anticipar cuando los pares de lengua son muy diferentes en cuanto al orden sintáctico. Es el caso entre el idioma alemán y el español, pues sabemos que el predicado verbal puede desvelarse al final de la oración en alemán, mientras que en el español se exige su presencia tras el sujeto. Esta diferencia estructural es la que fuerza al intérprete a anticipar verbo y sentido. Este tipo de anticipación forzosa u obligatoria, ha sido estudiada por diversos investigadores como Chernov $(1979,1981)$ o como Jones (1998) entre otros.

Esta sería una de las posibles explicaciones a la concurrencia de la anticipación en IS. Otra sería la de adelantar elementos no necesariamente verbales "justo antes de que lo verbalice el orador" (Liontou 2015: 15; Van Besien 1999: 250). Este efecto puede deberse al conocimiento lingüístico, situacional y extralingüístico del intérprete. Algunos investigadores consideran que es "una herramienta estratégica fundamental en el procesamiento del discurso" (Kohn y Kalina 1996: 124) ${ }^{1}$.

Nada de esto sería posible sin la redundancia presente en el lenguaje a la que Chernov $(1979,1981)$ confiere un papel nuclear en la ejecución de la IS. "El sentido, al contrario que el significado, solo se manifiesta a través de la predicación, de manera explícita o implícita" (Chernov 1979: 281); la

${ }^{1}$ (...) a fundamental feature of strategic discourse processing. 
iteración de elementos semánticos del discurso y el sentido explícito o implícito forman parte del eje en torno al cual explica el fenómeno de la anticipación, basándose en el modelo de predicción de probabilidades (Chernov 1979, 1981). Distingue entre la estructura superficial lingüística del discurso y la estructura semántica profunda, más difícil de comprender. Y concluye que aunque las palabras tengan significado, este significado no sirve para la interpretación, pues debe tener sentido dentro de la estructura semántica profunda del discurso

Cualquier intérprete aprende desde los inicios de su entrenamiento, que no son importantes las palabras, sino las ideas en el discurso y la conexión con su referente exterior. Eso es lo que da sentido al discurso y permite avanzar, incluso cuando se pierde información o se desconoce el significado concreto de algunas palabras o términos.

Esto explica que aun siendo la interpretación simultánea una actividad compleja desde el punto de vista cognitivo, haya miles de intérpretes a lo largo del mundo que demuestran, día a día, que es posible ejecutarla con un nivel de calidad profesional y experto.

Por lo tanto, la comprensión del discurso es vital para la interpretación, si no se comprende la conexión entre las diferentes unidades de sentido del mismo, el/la intérprete pierde visión de conjunto y de pronto cae en la cuenta de que está traduciendo palabras, pero no discurso. Esto se debe a que el/la intérprete se ha perdido algún elemento vital para la comprensión de la intención y la dirección hacia la que va el orador. Es decir, ha perdido la capacidad de anticipación de sentido y los árboles no le dejan ver el bosque.

La anticipación es lo que permite mirar más allá de las palabras y una vez comprendido el sentido, permite reformular el discurso. Es decir, es el elemento básico para la interpretación. No obstante, a pesar de confirmar su existencia, observamos que se suceden los intentos de explicar su aparición. Es lo que sucede con la definición que aporta Lintou (2015: 16) sobre este fenómeno en la Enciclopedia Routldege:

Chernov aplicó el principio de predicción probable especialmente al mecanismo de comprensión en interpretación simultánea, y Kalina (1998) cataloga la anticipación como una estrategia orientada a la comprensión, pero en un sentido más amplio debemos asumir que forma parte de cualquier proceso de comprensión basado en la experiencia y en el conocimiento del intérprete (Lintou 2015: 16)².

${ }^{2}$ Chernov applied the principle of probability prediction mainly to comprehension in SI, and Kalina (1998) lists anticipation as a comprehension oriented strategy in interpreting, but an- 
Nosotros consideramos que todas las explicaciones ofrecidas hasta ahora son válidas, abordan el fenómeno, pero se quedan incompletas. Desde nuestro punto de vista, y tras la investigación realizada para este artículo, hemos llegado a la conclusión de que no debe ser considerada ni catalogada como una estrategia, sino como un elemento omnipresente en el ser humano. Existe en cualquier actividad, especialmente, en la comunicación. Nos pasamos la vida o anticipando o prediciendo en nuestros quehaceres diarios, y el mundo no funcionaría bien sin esta facultad. Imaginemos algo tan normal como la conducción de un vehículo; todos los que sabemos conducir estamos internamente conectados con los movimientos de los coches que nos rodean, y podemos predecir si el conductor que tenemos delante, va a girar o va a frenar bruscamente o al menos nos permite activar un predicción de probabilidad de movimiento, tal y como planteaba Chernov (1981).

Esta capacidad anticipatoria, nos ha permitido sobrevivir como especie -y como intérpretes-, y se produce porque estamos diseñados biológicamente para mejorar nuestra capacidad de colaboración y de supervivencia. En este artículo veremos la importancia de esta facultad en interpretación simultánea y la relación que tiene con nuestra biología, relación que ya intuyó Chernov y la escuela rusa de sicología.

\section{La búsqueda de sentido en el discurso: lenguaje natural versus lenguaje escrito}

Para comprender el sentido de un discurso, el ser humano activa durante el proceso comunicativo un mecanismo que consiste en la búsqueda constante de sentido en todo lo que lee o escucha - search after meaning (Graesser et al. 1994, citado en Hild 2015: 69). La interpretación simultánea es una actividad comunicativa algo especial, pues se produce en un marco poco natural. Existe un emisor, un mensaje, un canal de comunicación y un receptor final. Para que el mensaje, en este caso el discurso, despliegue todo su contenido y su sentido, es imprescindible que el profesional se documente previamente; debe dominar la lengua con el registro, la fraseología, el léxico y la terminología propia de la materia de la que se trate. Es decir, que esta búsqueda de sentido por parte de lo que Graesser llama the comprehender - estará fuertemente limitada por todo este trabajo anterior.

ticipation in the broader sense must be assumed to be part of any process of understanding based on prior experience and knowledge. 
Esto es así porque no se trata de un contexto comunicativo informal, sino claramente estructurado, que se realiza en el marco específico de un congreso o de una institución u organismo determinado. La documentación y preparación previas, la interacción con la lengua en uso, así como el conocimiento previo sobre la materia (Zwaan y Rapp 2006, citados por Hild 2015: 69) intervienen para poder actuar en este acto comunicativo, y en un concepto más amplio estarían las cuatro áreas definidas por Shreve (2006, citado en Alves 2015: 26) relativas al (1) conocimiento lingüístico de L1 y L2; (2) el de la cultura de partida y de llegada; (3) estrategias de traducción y (4) herramientas para buscar información. Estas cuatro áreas se refieren al ámbito de la traducción, pero son extrapolables al de la interpretación.

Como decíamos, el marco comunicativo en el que se produce la interacción es un tanto artificial, está jerarquizado y, además, depende de la tecnología para que pueda ejecutarse. El sonido no llegará al intérprete de manera natural, sino a través de unos aparatos especiales; si no puede ver al orador y este efectúa algún gesto de lenguaje no verbal, el intérprete no podrá desambiguar el mensaje. Todo este conjunto de circunstancias, propias de la interpretación simultánea, afecta al grado de anticipación habitual que tiene lugar en cualquier situación comunicativa. Estas circunstancias afectan de lleno a la redundancia omnipresente en el lenguaje natural y al modelo de predicción de probabilidades postulado por Chernov. En su investigación sobre la importancia de los elementos semánticos y el sentido del discurso, decía lo siguiente:

Lo esencial para un intérprete es no perder el hilo mental de las relaciones entre los componentes semánticos y transferirlos lo más certeramente posible, dado que a ellos se debe que aflore el sentido de entre el cúmulo de componentes semánticos que aporta la lengua natural y que son activados en el discurso (Chernov 1979: 288)3.

Chernov habla de ese "cúmulo de componentes semánticos que aporta la lengua natural", ahora bien, ¿es lengua natural lo que se habla en un congreso? Analicemos el tipo de discurso que se tenga que interpretar y el marco comunicativo en el que se produce. En el caso de la interpretación simultánea el emisor es un orador, que puede conocer o no su oficio y por ende complicar

${ }^{3}$ The important thing for the interpreter is to keep mental track of, and transfer as accurately as posible, the relations of semantic components, since it is they that make for the appearance of sense out of the stock of semantic components provided by natural language and activated in discourse. 
la ejecución de una interpretación por muy profesional que sea el intérprete. El orador puede expresarse de un modo próximo a la comunicación natural, con un lenguaje libre y casual y hablarnos de su experiencia personal, lo que todos sabemos que facilita la interpretación; o bien se puede dirigir a la audiencia con un texto escrito que, con suerte, lleva las suficientes marcas de oralidad que permitan su comprensión; o por el contrario se trata de un "orador-lector", es decir, un conferenciante que ha redactado un discurso denso y cargado de terminología, o puede ser un conferenciante incapaz de ofrecer un mensaje estructurado; o puede tratarse de alguien que no vocaliza, que lee a toda velocidad, etc. ... Son muchos los factores que influyen en la calidad de la interpretación, justamente, porque aun siendo un marco comunicativo, no es todo lo natural que debiera ser.

Como hemos dicho, el conferenciante suele haberse preparado el discurso de antemano y esta preparación previa exige una versión escrita para poder leer a la audiencia, aunque luego se aligere con algunas anécdotas o expresiones más personales y libres por parte del orador. Ahora bien, un texto escrito no es el resultado de un lenguaje natural y casual. Escribir o leer no son cualidades espontáneas ni innatas en el desarrollo normal de un ser humano. El ser humano aprende a hablar y a caminar, porque biológicamente está diseñado para que estas competencias se desarrollen. Pero la lectura y la escritura, no son competencias espontáneas y naturales. El dominio de la escritura como medio de comunicación y la alfabetización exigen años de trabajo (Li \& Hombert 2002: 198). No saber leer o escribir, sigue siendo una realidad que afecta a la adquisición de conocimiento, pero no se es más o menos inteligente por ello.

En un texto escrito no existen elementos paralingüísticos, gesticulación corporal o facial, ni tonos que permitan intuir que estamos en un marco comunicativo natural. Por lo tanto, la interpretación del sentido en un texto escrito exige un conocimiento previo de convenciones textuales y de conocimiento cultural o particular. Eso permite al lector saber en dónde está y hacia dónde se dirige el sentido del texto. La ficción y la creatividad del autor suponen además un reto para un lector no experimentado, pensemos en lo que cuesta enseñar a leer a adolescentes en los colegios. Leer y escribir, por lo tanto, no son facultades innatas en el ser humano; hablar, sí.

En los casos en los que el marco natural de comunicación es inexistente, la anticipación que, como explicaremos, está presente en cualquier ámbito comunicativo o quehacer humano, deja de activarse de manera natural; hay que forzarla, hay que entrenar para conseguir seguir al orador en su campo. Esto es así, porque, tal y como hemos dicho, el lenguaje ha dejado de 
ser natural, está basado en un discurso, y este discurso estará escrito, lo cual resta oralidad y naturalidad a la lengua, y reduce la espontaneidad, y la anticipación también innata en la biología del ser humano. Entonces, hay que contrarrestar esta artificialidad que entorpece la naturalidad del discurso y la anticipación. Pero, ¿se puede?

\section{Aprender y enseñar a anticipar}

Todos los autores (Gillies 2013; Shrieve 2006) coinciden en afirmar que hay que estudiar e investigar sobre la materia de la que debamos interpretar. Esta es, hasta la fecha, la única forma de entrenar el mecanismo de anticipación sobre temáticas de las que se desconoce todo, por no ser especialistas. Esta preparación ayuda a eliminar el factor sorpresa, elementos sorpresa según la terminología de Gillies (2013: 214). ¿En qué consisten estos elementos sorpresa? Habitualmente no se debe a la aparición de una palabra desconocida, pues cuando esto sucede el intérprete puede continuar su actividad omitiendo la misma, aprovechando la redundancia presente en el lenguaje, siempre y cuando este vocablo concreto no este cargado de sentido específico, pues de tenerlo no se trataría de una palabra cualquiera que podría omitirse sin más, sino de un término que aporta sentido a la materia y a la línea argumental. La consecuencia es que el intérprete pierde la línea argumental.

El mismo efecto se produce cuando el orador inicia una línea argumental nueva y sorpresiva. El intérprete había sido capaz de estar anticipando sentido, gracias a la preparación y documentación previas. Pero, de pronto, surge algo inesperado, casi inconexo con el sentido y los argumentos de lo precedente. Aquí también se produce un factor sorpresa que genera estrés y que interrumpe el mecanismo de anticipación interna que estaba activado y permitía al intérprete reformular adecuadamente. Estos contratiempos suceden todos los días al profesional de la interpretación, pero consigue salir adelante sin que se note excesivamente el bache en el discurso.

Ahora bien, esta preparación previa ayuda a interpretar porque el profesional, poco más o menos, se ha convertido en un especialista de la materia. Un especialista muy sui generis, pues no tendrá un conocimiento excesivamente profundo, pero lo bastante adecuado como para entender las líneas argumentales que son las que guían el discurso. Ello no evitará que haya un porcentaje de elementos sorpresa, pero este porcentaje será menor con preparación que sin ella. Por lo tanto, entendemos que los ejercicios de cloze o de práctica de la anticipación (Kalina 1992, 2000;) que se proponen y se 
practican en las clases, son adecuados para los estudiantes de interpretación. Como parte integrante se suele proponer que el estudiante aprenda a realizar discursos (Gillies 2013). Esta actividad discursiva es, indiscutiblemente, buena y adecuada para los alumnos, porque les enseña a estructurar y a ordenar ideas, para su correcta exposición. Además, al colocarse en el papel de oradores aprenden a qué velocidad deben hablar y de qué cosas y con qué densidad informativa, para que sus compañeros que están ejecutando la práctica de interpretación, puedan efectuar una interpretación adecuada. Este intercambio de papeles es muy productivo en clase.

Ahora bien, como decía Gillies (2013: 214) "las convenciones para escribir discursos no son muchas, están limitadas” y además (Gillies 2005, citado en Gillies 2013: 216) "aprender a escribir un discurso ayuda a anticipar lo que el orador está a punto de decir". Esta clase de entrenamiento en la oratoria, que es de tipo intelectual, también es comparable al entrenamiento deportivo. A pesar de que pueda parecer que los músculos y el cerebro van por caminos diferentes, esto no es así. Cuestión de la que hablaremos algo más adelante. Ponerse en la piel de un orador y practicar las convenciones textuales de redacción de tipos de discurso diferentes, enseña a los estudiantes a incorporar dos aspectos fundamentales para su posterior actividad profesional: la estructura o esquema de redacción de un discurso y las ideas y materiales que son los elementos intangibles que están empujando el texto en un sentido determinado. Es decir, habrá una estructura digamos superficial, idéntica en cada una de las modalidades textuales, y otra más profunda que afecta al mensaje.

Con estos ejercicios se consigue que los alumnos incorporen a su acervo lingüístico cognitivo un objeto nuevo: el discurso y sus formas. Al igual que se hace cuando se aprende la lista de verbos irregulares o la conjugación, o la flexión de los diferentes elementos que ayudan a utilizar la lengua en su versión escrita, la estructura discursiva se añade como un objeto más a ese conocimiento que vamos integrando en nuestro cerebro. Es como un libro más que se añade a nuestra biblioteca del saber. El lenguaje no creativo, funciona con convenciones textuales, igual que patrones de costura. Siempre son las mismas, permite saber por dónde irán las costuras y por lo tanto la anticipación está presente durante todo el proceso, porque es el resultado del modelo de predicción de la probabilidad, modelo que hemos incorporado a base de aprender discursos similares y de estudiar las líneas argumentales posibles

${ }^{4}$ Knowing how to build up (create) a speech yourself will mean you'll find it easier to anticipate what a speaker is going to say. 
y probables. Esto se complica cuando el lenguaje que usamos es creativo y no imitativo, pues las pautas no existen.

Todos estos ejercicios dejan una huella cognitiva en nuestro cerebro, un patrón neuronal que se almacena y queda silente, hasta que se escucha un discurso que nuestro almacén cognitivo reconoce. Entonces se activa el mecanismo y nos permite seguir los pasos al orador.

Ericsson (2000) investigó la excelencia profesional en interpretación. Se fijó en experimentos realizados con anterioridad para demostrar que a la excelencia solo se puede llegar gracias a la práctica deliberada. Si un músico o un ajedrecista querían mejorar hasta el punto de llegar a la excelencia profesional, se percató de que el entrenamiento debía ser individual y con especial atención a los fallos o defectos que debían eliminar en su ejecución musical o en su juego. Se conseguía con la práctica deliberada consciente para superar esos fallos. Los ajedrecistas lo hacían estudiando partidas de otros, incorporando de este modo, ese programa neuronal que les permitía reconocer una tipo de estrategia y adelantar posibles jugadas para permitirles poner en práctica lo que Chernov (1981:31) denominó "modelo de predicción de probabilidades". Aprender estrategias y partidas, es similar a redactar discursos. Quedan incorporadas, como un aprendizaje más en nuestro cerebro y permite reconocer un tipo de estrategia discursiva o ajedrecista determinadas.

Cuando Ericsson estudió la manera en que los mecanógrafos practicaban para mejorar su velocidad de escritura, se dio cuenta de lo siguiente: si se les permitía teclear e ir leyendo lo que aún no se había tecleado, la velocidad mejoraba considerablemente. Ahora bien, cuando se les tapaba el resto del texto y no podían mirar más allá de la palabra que estaban tecleando en cada momento, la velocidad no mejoraba. Es decir, los sujetos que intervenían en el experimento, de hecho estaban anticipando el mensaje y eso les permitía alcanzar la excelencia profesional. Concluyó constatando que este grado de excelencia

(...) no ocurre de manera automática ni por arte de magia. La mejora en la ejecución está ligada a cambios en los mecanismos cognitivos que intervienen en la manera en que el cerebro y el sistema nervioso controlan esta ejecución y tiene que ver con el grado de adaptación de los sistemas fisiológicos del cuerpo (Ericsson 2000: 202$)^{5}$.

${ }^{5}(\ldots)$ adult experts doesn't happen automatically nor magically. Performance improvement can be linked to changes in cognitive mechanisms mediating how the brain and nervous system control performance and in the degree of adaptation of physiological systems of the body. 
De este experimento destacamos ese "mirar más allá de la palabra tecleada" y lo destacamos porque tiene, indiscutiblemente, mucho que ver con interpretación. Uno de los elementos fundamentales en el aprendizaje de la interpretación simultánea es la traducción a la vista. Se trata, como sabemos, de traducir un texto con apenas preparación previa. Se debe realizar procurando mantener la fluidez y la naturalidad de lectura, como si se tratara de un texto no escrito en un idioma extranjero. Curiosamente, para conseguir realizar este ejercicio es necesario mantener el cerebro ejecutando dos acciones al mismo tiempo: reformular y avanzar en la lectura del texto para incorporar la nueva información e insertándola en el discurso. Los ojos del intérprete buscan en el texto el material clave que le permita anticipar, es decir, comprender, para de esta forma poder reformular. Si no sabe cómo continúa la frase o el párrafo, no sabrá como ordenar adecuadamente y con los parámetros de calidad exigidos, el texto que está traduciendo en voz alta.

En realidad, es un ejercicio necesario para la simultánea, porque sabemos que dependemos del material que aún no se ha interpretado para poder ordenar la sintaxis de nuestros enunciados y la coherencia del discurso que estamos interpretando. Los mecanógrafos pueden trabajar al máximo de su concentración para alcanzar la velocidad perfecta, durante 15 o 30 minutos. Y además esto les permite anticipar (Ericsson 2000: 201) ${ }^{6}$. Casualmente, este es también aproximadamente el tiempo recomendable de trabajo para cada uno de los profesionales que se van alternando en la interpretación.

\section{Neuronas espejo y anticipación}

Tanto Chernov como Ericsson intuían la presencia de algún tipo de mecanismo cognitivo que permitía alcanzar el nivel de excelencia.

En realidad, aún no se había descubierto la existencia de las neuronas espejo (Rizzolatti et al. 1996). Estas neuronas pertenecen al sistema visomotórico del ser humano, es decir, se activan con la ejecución del movimiento, pero también con la visualización u observación del mismo. Y esto resulta de sumo interés en todas las actividades humanas, también en la interpretación.

${ }^{6}$ While straining themselves to type at a faster speed (typically around $10-20 \%$ faster than their normal speed) typists seem to strive to anticipate better - possibly by extending their gaze ahead further. 
Resulta imprescindible comprender al ser humano en su biología, porque cuanto más conozcamos los elementos que entran en juego en el aprendizaje, más capacitados estaremos para diseñar modelos eficaces de enseñanza. En particular, algunos autores intuyen que deben haber procesos neuronales que participan en el aprendizaje de alguna manera especial.

Rizzolatti et al. (2002) descubrieron que cuando se ejecutaba una acción, este acto físico dejaba rastro neuronal en el cerebro. Pero no sólo sucedía al realizar verdaderamente la acción, sino también por la simple observación esta se copiaba internamente. Este rastro neuronal al estilo de una pauta o un esquema determinado específico para cada tipo de acto, es lo que permite reconocer ese acto cuando lo va a ejecutar otra persona. Es decir, el ser humano es capaz de identificar la intención o el sentido de un movimiento físico en otras personas, porque reconoce su existencia dentro de su propia experiencia. En otras palabras, podemos adivinar o anticipar como va concluir una acción o un movimiento, por el simple hecho de que existe un conocimiento previo, y este puede haber sido ejecutado por la persona o simplemente observado, porque deja el mismo rastro neuronal. Rizzolatti lo explicaba de esta manera:

Como base de nuestra hipótesis asumimos que el sistema de neuronas espejo que permite a los seres humanos comprender una acción es que el patrón neuronal que se activa de forma endógena para activar la ejecución de esa acción, es el mismo que se activa de forma exógena con la mera observación de la acción. Por lo tanto, si uno acepta que el individuo que va a ejecutar una acción "sabe" cuál va a ser el resultado de su acción, entonces debemos concluir que ese sujeto también será capaz de saber el resultado de una acción que no esté ejecutando, sino observando, porque es el mismo mecanismo el que actúa en ambos casos. (Rizzolatti et al. 2002: 51) ${ }^{7}$

¿Qué nos revelan estos datos? En realidad, lo que interesaba a los investigadores era averiguar si el sistema humano de neuronas espejos tenía o no "el potencial de explicar cuál era el mecanismo neurofisiológico, responsable de la mecánica de la imitación en los seres humanos" (Rizzolatti et al. 2002: 55) ${ }^{8}$.

7 The assumption at the basis of the hypothesis that mirror system allows action understanding is that the same neuronal pattern that is endogenously activated for action execution is also activated exogenously during action observation. Thus, if one accepts the notion that the acting individual "knows" what will be the results of his/her action, one has to admit also that he/ she will be able to know the outcome of the observed action, being the same mechanism involved in both occasions.

${ }^{8}$ In conclusion, although at this stage all these hypotheses lack of experimental support, 
Esta capacidad imitativa del ser humano es clave en el aprendizaje humano, en general, pero también de lenguas.

\section{Cuerpo y mente}

Todo este sistema de neuronas espejo sigue investigándose para conocer su implicación en muchas de las facultades y competencias de los seres humanos. Por lo expuesto hasta el momento, parecería que estamos hablando exclusivamente de movimiento y de acciones, más físicas que mentales. Pero poco a poco iremos comprendiendo que lo físico no está alejado de lo mental.

Esa capacidad que tenemos de anticipar el sentido de un movimiento, quedó de manifiesto en uno de los múltiples experimentos que se han llevado a cabo desde el descubrimiento de las neuronas espejo. En uno de ellos (Becchio et al. 2012) demostraron que dicha facultad existía. Cuando se colocaba a unas personas como agentes y a otras como observadoras de la ejecución de una acción que se dejaba inconclusa, los observadores eran capaces de adivinar, es decir, anticipar el sentido o la intención de la acción.

Esto no debería sorprendernos demasiado, pues muchas de las actividades físicas desarrolladas por el ser humano desde tiempos prehistóricos hasta el día de hoy serían inimaginables, sin esta capacidad de anticipación. Pero, sin remontarnos muy atrás, debemos ser conscientes de que toda actividad en la que intervienen más de dos personas, es una actividad compleja (como la interpretación simultánea) que sería imposible de ejecutar sin este mecanismo de predicción. Pensemos en el fútbol, en el baloncesto, en cualquier deporte, o incluso en aquellas actividades que exigen la colaboración de otros. Si los jugadores no tuvieran esa capacidad de adivinar las intenciones de los jugadores del equipo rival, sería un auténtico desastre. Los participantes en deportes que se juegan en equipo, son capaces de prever las acciones de los jugadores contrarios o al menos de ejecutar hipótesis predictivas sobre sus intenciones.

Para poder participar en actividades interactivas como desplazar una mesa entre varias personas, bailar el vals o jugar al fútbol, los intervinientes deberán ser capaces de ajustar sus acciones a las de la otra persona, y ejecutar una acción complementaria de manera apropiada y a tiempo. Eso requiere de la habilidad de predecir el siguiente paso que el otro va a dar, y también de la

a mechanism with the characteristic of the mirror system appears to have the potentiality to give a neurophysiological, mechanistic explanation of imitation (Rizzolatti et al. 2002: 55). 
habilidad de saber cuándo se van a realizar esas acciones; es decir, que deberán generar predicciones sobre la velocidad de actos de los demás (Sebanz \& Knoblich 2009, citado en Manera et al. 2013: 5)9.

Todo esto es posible gracias a las neuronas espejo que, como hemos dicho, forman parte del sistema visomotórico y se activan con la ejecución y con la observación del movimiento. Ahora bien, también se ha descubierto que están presentes "en el área de Broca, un área responsable de la lengua pero también de los movimientos de la mano"10 (Binkofski et al. 1999, citado en Rizzolatti et al. 2001: 42). Un área en la que parece que se mezclan lengua y movimiento de manos. Dos aspectos presentes en la lengua de signos de los sordomudos, pero también en la gesticulación durante la interpretación simultánea.

La neurociencia se refiere a todos estos procesos con el nombre de programas neuronales que permiten entender, copiar, imitar ejecutar una acción o incluso aprender un idioma. Puede ser que esta misma pauta sea la que permita a un intérprete realizar con éxito su cometido. En realidad, la anticipación no es una estrategia en interpretación simultánea. Una estrategia consiste en una serie de acciones pensadas, y diseñadas conscientemente, para alcanzar un objetivo concreto. Un intérprete sólo puede utilizar una estrategia para intentar predecir o anticipar sentido, y esa estrategia consiste en desarrollar un rastro neuronal interno que le permita rastrear el sentido del texto o la intención del orador, como un auténtico detective que sigue una pista. Esa estrategia es la documentación, la preparación, porque son actividades que dejan huella neuronal, pautas y patrones, como convenciones textuales en un discurso, que permiten identificar o reconocer como propias las palabras ajenas. Igual que un entrenamiento, en el que aparte de entrenar físicamente, se muestran las estrategias y se analiza el juego del adversario para reconocer el siguiente movimiento, un intérprete solo es capaz de anticipar si convierte en experiencia propia o ajena, lo que está a punto de interpretar. Sin conocimiento no se genera experiencia (Alves 2015: 20) ${ }^{11}$, hace falta practicar y al igual que en deporte.

9 To successfully engage in interactive activities such as carrying a table together, dancing a waltz, or playing football, actors must be able to adjust their actions to those of the other person, choosing an appropriate complementary action to be performed at an appropriate time. This requires the ability to predict what others will do next, but also when their actions will take place, i.e., to generate predictions about the timing of others' actions.

${ }^{10}$ This last inference was recently strongly corroborated by findings showing that Broca's area is an area in which not only speech but also hand movements are represented.

${ }^{11}$ Learning is consolidated by experience. 
La interpretación, a pesar de que se enseña en las universidades, no es únicamente una asignatura académica; es más bien algo parecida a una arte o a un deporte. Uno no puede aprender a interpretar asistiendo a una lección magistral (o leyendo un libro) en que se explica cómo funciona la interpretación ${ }^{12}$ (Gillies 2013: 3).

Al igual que sucede con cualquier disciplina deportiva, uno tiene que practicar y no sirve de mucho leer cómo convertirse en un deportista. Pero llegado a este punto, habría que distinguir entre dos planos de una actividad. Un plano sería la fuerza muscular precisa que se puede adquirir con un adecuado entrenamiento físico, y el otro es la mente del deportista. La fuerza física es importante, pero lo es aún más la resiliencia y la acción positiva. Todo eso sucede en el cerebro y convierte a un deportista en uno de élite o del montón. Tiene que ver con patrones neuronales que se han ido desarrollando con un entrenamiento adecuado. Si retomamos el ejemplo del ajedrecista que estudia las partidas de otros campeones, se comprenderá que lo que ha hecho es visualizar internamente las diferentes estrategias, como si las hubiera vivido en primera persona. Como ya dijimos, eso le permitirá identificarlas, cuando le sucedan en una partida real.

Es decir, que el entrenamiento deportivo es tanto físico como mental: se observan los movimientos de otros para identificar las estrategias que ayuden a reconocer la intención del contrario. En este sentido, el deporte tiene algo en común con la interpretación. Un intérprete aprende a identificar estructuras y elementos en los discursos, porque se prepara con una documentación adecuada, lo que se convierte en experiencia. Al igual que internalizar las partidas de ajedrez se convierte en experiencia para el cerebro del ajedrecista, así el estudio de documentación y la práctica generan experiencia en el cerebro del intérprete. Este hecho queda de relieve en el libro de Gillies con la respuesta que dio un intérprete profesional, acerca del desfase entre el inicio de la interpretación y el discurso del orador:

Estudiante intérprete: ¿Considera que con los años de experiencia el desfase entre el orador y su interpretación es cada vez mayor?

Intérprete experimentado: En realidad, no es así. Ahora siento que puedo anticipar lo que se va a decir con cada vez mayor precisión (Gillies 2013: 213).

${ }^{12}$ Interpreting, despite the fact that it is often taught at universities, is not an academic subject: it is far more akin to a craft or a sport. One cannot learn to interpret by going to a lecture (or reading a book) and understanding an explanation of how interpreting works. 


\section{Conclusiones}

El descubrimiento de las neuronas espejo ha abierto un nuevo campo a la neurociencia, la traductología, a la psicolingüística, a la antropología, etc. Su implicación en la transmisión de cultura, aprendizaje de lenguas, empatía, imitación y anticipación, por mencionar algunas funciones destacadas, permite a los investigadores explicar desde un nuevo enfoque, el potencial de aprendizaje del ser humano.

La interpretación simultánea y el proceso de anticipación es uno de ellos. Todos los profesionales constatan que existe y que facilita enormemente la tarea al intérprete. La neurociencia supone un paso más en la transversalidad con la que se pueden abarcar desde múltiples perspectivas una actividad humana. Aunque las investigaciones estén aun en sus comienzos, ya están aportando luz acerca de fenómenos como el de la empatía que permite a los intérpretes reformular con mayor precisión y anticipar con mayor seguridad. Entendiendo que empatía es la capacidad de identificar intenciones y emociones en el otro como propias y que se reconocen como experiencias compartidas.

Morrisson (2002: 337-338) ${ }^{13}$ se refería a este aspecto de las emociones:

La emoción influye en el comportamiento futuro, porque empareja la percepción en sí con una disposición a actuar (véase Le Doux 1998). (...) Esto significa que la disposición a actuar se recuerda mejor como consecuencia de la fuerza y el carácter emocional de la respuesta que indujo a actuar.

Las neuronas espejo están permitiendo avances en el campo de la inteligencia artificial y del aprendizaje de lenguas. Nos ayudan a comprender la manera en que formamos representaciones mentales con la simple observación (Decety 2001). Aún queda mucho por investigar, pero el futuro es prometedor para la interpretación simultánea y la anticipación como proceso presente en su ejecución.

\section{Referencias bibliográficas}

Alves F., 2015, Translation Process Research at the Interface. Paradigmatic, Theoretical, and Methodological Issues in Dialogue with Cognitive

${ }^{13}$ Emotion influences future behaviour by coupling perception with disposition to act (e.g. LeDoux 1998). (...) This means that certain action dispositions become better remembered by virtue of the strength and character of the emotional response that produced them. 
Science, Expertise Studies, and Psycholinguistics, [in:] Psycholinguistic and Cognitive Inquiries into Translation and Interpreting, A. Ferreira, J. W. Schwieter (eds.), Amsterdam-Philadelphia, pp. 17-40.

Bartłomiejczyk M., 2008, Anticipation: A Controversial Interpreting Strategy, [in:] Translation and Meaning Part 8, M. Thelen, B. Lewandowska-Tomaszczyk (eds.), Maastricht, pp. 117-126.

Besien F. van, 1999, Anticipation in Simultaneous Interpreting, "Meta", Vol. 44(2), pp. 250-259.

Becchio C., Manera V., Sartori L., Cavallo A., Castiello U., 2012, Grasping intentions: From thought experiments to empirical evidence, "Front Hum Neurosci", Vol. 6, https://www.ncbi.nlm.nih.gov/pmc/articles/ PMC3340947/ (access: 8.01.2019).

Binkofski F. et al., 1999, A Fronto-Parietal Circuit for Object Manipulation in Man: Evidence from an fMRI-Study, [in:] Mirror Neurons and the Evolution of Brain and Language, Amsterdam-Philadelphia, pp. 37-59 .

Chernov G. V., 1979, Semantic Aspects of Psycholinguistic Research in Simultaneous Interpretation, "Language and Speech", Vol. 22(3), pp. 277-295 .

Chernov G. V., 1981, "Semantic Redundancy as a Key to Reliable Comprehension of a Verbal Message (Objective and Subjective Factors)", [in:] Comprehendre le Langage: Actes du Colloque International et Multidisciplinaire sur la Comprehénsion du Langage, Didier Collection "Linguistique" $\mathrm{n}^{\circ} 12$, Paris, pp. 31-37.

Decety J., 2001, "Is there such a Thing as Functional Equivalence between Imagined, Observed, and Executed Action?", [in:] The imitative Mind. Development, Evolution, and Brain Bases, A. N. Meltzoff, W. Prinz (eds.), Cambridge University Press, Cambridge-New York-Melbourne-Madrid-Cape Town-Singapore-Sao Paulo, pp. 291-310.

Ericsson K. A., 2000, Expertise in Interpreting, "Interpreting", Vol. 5(2), pp. 187-220.

Ferreira A., Schwieter J. W. (eds.), 2015, Psycholinguistic and Cognitive Inquiries into Translation and Interpreting, Amsterdam-Philadelphia.

Gile D., 2015, "The Contributions of Cognitive Psychology and Psycholinguistics To Conference Interpreting. A Critical Analysis", [in:] Psycholinguistic and Cognitive Inquiries into Translation and Interpreting, A. Ferreira, J. W. Schwieter (eds.), Amsterdam-Philadelphia, pp. 41-64. Gillies A., 2013, Conference Interpreting. A Student's Practice Book, New York. 
Hild A., 2015, "Discourse Comprehension in Simultaneous Interpreting. The role of Expertise and Information Redundancy", [in:] Psycholinguistic and Cognitive Inquiries into Translation and Interpreting, A. Ferreira, J. W. Schwieter (eds.), Amsterdam-Philadelphia, pp. 67-100.

Jones R., 1998, Conference Interpreting Explained, St. Jerome Publishing, Manchester.

Kohn K. \& Kalina S., 1996, The Strategic Dimension of Interpreting, "Meta", Vol. 41(1), pp. 118-138.

LeDoux J., 1998, The Emotional Brain, [in:] Mirror Neurons and the Evolution of Brain and Language, V. Gallese, M.I. Stamenov (eds.), Amsterdam-Philadelphia, pp. 333-340.

Li C.N. \& Hombert J. M., 2002, On the Evolutionary Origin of Language, [in:] Mirror Neurons and the Evolution of Brain and Language, V. Gallese, M. I. Stamenov (eds.), Amsterdam-Philadelphia, pp. 175-205.

Lintou K., 2015, Anticipation, [in:] Routledge Encyclopedia of Interpreting Studies, F. Pöchhacker (ed.), London, pp. 15-16.

Manera V. et al., 2013, Time will show: Real Time Prediction during Interpersonal Action Perception, "PLOS ONE", Vol. 1: e54949, pp. 1-6.

Meltzoff N. \& Prinz W. (eds.), 2001, The imitative Mind. Development, Evolution, and Brain Bases, Cambridge-New York-Melbourne-Madrid-Cape Town-Singapo -Sao Paulo.

Morrison I., 2002, Mirror Neurons and Cultural Transmission, [in:] Mirror Neurons and the Evolution of Brain and Language, V. Gallese, M. I. Stamenov (eds.), Amsterdam-Philadelphia, pp. 333-340.

Rizzolatti G. et al., 2001, From Mirror Neurons to Imitation: Facts and Speculations, [in:] The imitative Mind. Development, Evolution, and Brain Bases, A. N. Meltzoff, W. Prinz (eds.), Cambridge University Press, Cambridge - New York - Melbourne - Madrid - Cape Town - Singapore Sao Paulo, pp. 247-290.

Rizzolatti G. et al., 2002, The Mirror System in Humans, [in:] Mirror Neurons and the Evolution of Brain and Language, V. Gallese, M. I. Stamenov (eds.), Amsterdam-Philadelphia, pp. 37-59.

Sebanz N., Knoblich G. (2009), Prediction in Joint Action: What, When, and Where, "Topics in Cognitive Science", Vol. 1(2), pp. 353-367.

Stamenov M. I. \& Gallese V. (eds.), 2002, Mirror Neurons and the Evolution of Brain and Language, Amsterdam-Philadelphia.

Womble S., \& Wermter S., 2002, Mirror Neurons and Feedback Learning, [in:] Mirror Neurons and the Evolution of Brain and Language, V. Gallese, M. I. Stamenov (eds.), Amsterdam-Philadelphia, pp. 353-362. 


\section{Mirror neurons and anticipation in simultaneous interpreting Summary}

Anticipation is a frequent phenomenon in simultaneous interpreting [SI]. Some authors argue (Gile, 1995; Chernov 1994, 2004) that it is compulsory due to the structural differences in syntactical order between the pair of languages the interpreter is working with. Other researchers (Chernov 1979, 1981; Jones 1998) refer to it as a strategy in simultaneous interpreting. In our view, anticipation cannot be considered a strategy (Kohn \& Kalina, 1996) that the interpreter switches on or off in SI. Our claim is that it cannot be switched off. Anticipation is the basis of reformulation itself. If the interpreter cannot predict the sense before the utterance has finished, it would be very difficult to reorganise or reformulate an utterance and render speech, words, syntax and sense.

Translation studies have broadened their scope including methodology in their research, and recently look to areas such as cognitive science or psycholinguistics (Ferreira, A. \& Schwieter, J.W., 2015). This interdisciplinary approach can shed some light over the processes present during translation or interpreting. One of these interdisciplinary approaches is neuroscience and the recent discoveries about the functions of the mirror neurons (Rizzolatti et al. 1996). Mirror neurons can help explain why there is a constant and natural anticipation process in human communication. Although these cells belong to the visuomotor system, some researchers acknowledge their share in cultural transmission, language acquisition and learning through observation.

Chernov $(1979,1981)$ advanced his theory of probability prediction model based on the presence of redundancy in natural language. But anticipation is present in normal and casual language as well (Li \& Hombert 2002) and that is perhaps the reason why it can help predicting intentions in a given communicative situation, just as Chernov postulated. Simultaneous interpreting is a very special communicative setting and language in a conference setting can hardly be considered casual or natural. It is mostly written language, with some exceptions, transmitted orally. This hampers the natural process of anticipation present in casual language or in natural communicative situations, which is why it has to be trained through the learning of material and documentation (Kalina, 1992, 2000; Gillies, 2013; Shrieve, 2006). This learning, on the other hand, also activates the mirror neuron system in the interpreter and leaves traces or neural patterns (Rizzolatti et al. 2002) that help again in the anticipation process.

Keywords: anticipation, mirror neurons, simultaneous interpreting

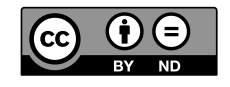

